\title{
Core promoters are predicted by their distinct physicochemical properties in the genome of Plasmodium falciparum Kevin Brick*, Junichi Watanabe ${ }^{\dagger}$ and Elisabetta Pizzi*
}

Addresses: *Dipartimento di Malattie Infettive, Parassitarie ed Immunomediate - Istituto Superiore di Sanità, Viale Regina Elena, 299, o0161 Rome, Italy. 'Department of Parasitology, Institute of Medical Science, The University of Tokyo 4-6-1, Shirokanedai, Minatoku, Tokyo 1088639, Japan.

Correspondence: Kevin Brick. Email: kevbrick@gmail.com

Published: 18 December 2008

Genome Biology 2008, 9:RI78 (doi:10.1 186/gb-2008-9-12-r178)

The electronic version of this article is the complete one and can be found online at http://genomebiology.com/2008/9/I2/R I78
Received: 26 August 2008

Revised: 3 November 2008

Accepted: 18 December 2008

(C) 2009 Brick et al.; licensee BioMed Central Ltd.

This is an open access article distributed under the terms of the Creative Commons Attribution License (http://creativecommons.org/licenses/by/2.0), which permits unrestricted use, distribution, and reproduction in any medium, provided the original work is properly cited.

\begin{abstract}
Little is known about the structure and distinguishing features of core promoters in Plasmodium falciparum. In this work, we describe the first method to computationally identify core promoters in this AT-rich genome. This prediction algorithm uses solely DNA physicochemical properties as descriptors. Our results add to a growing body of evidence that a physicochemical code for eukaryotic genomes plays a crucial role in core promoter recognition.
\end{abstract}

\section{Background}

Eukaryotic promoters are defined as regions containing the elements necessary to control the transcriptional regulation of genes. Typically, a promoter is organized into three regions. The core promoter (CP) spans the region approximately 35 bp upstream of the transcription start site (TSS) and is the binding region for the transcription initiation complex; the proximal promoter, which may contain several transcription factor binding sites, can range for hundreds of base pairs upstream of the TSS; finally, the distal promoter, which may contain additional regulatory elements, such as enhancers and/or silencers, can be located thousands of base pairs from the TSS. The best studied features of the canonical CP are proximal cis-acting sequence elements, which have been very well characterized in many organisms. These may include a TATA box, an Initiator element (Inr), a TFIIB recognition element (BRE), and a downstream promoter element (DPE). These sequence elements are, however, by no means ubiquitous, and in fact, it was recently estimated that only a maximum of $20 \%$ of mammalian promoters contain a TATA box $[1,2]$.
Much evidence has now emerged showing that epigenetic factors also contribute to transcriptional control of eukaryotic genes [3]. The term epigenetic has been redefined in a modern context as "the structural adaptation of chromosomal regions so as to register, signal or perpetuate altered activity states" [4]. Until recently, it has been difficult to computationally derive these structural adaptations from the DNA sequence; however, the recent work of Segal et al. [5] points to the existence of a periodic di-nucleotide 'code' that correlates strongly with nucleosome binding affinity. Interestingly, by using this 'code', it has been shown that nucleosome occupancy at TSS positions in human CPs is very low. Coming at this issue from another angle, it was recently shown that experimentally calculated DNA bendability and a penta-/ tetramer based compositional property of DNA exhibit characteristic profiles in the region of TSSs in several higher eukaryotes [6]. These distinctive changes in the conformational profile of DNA around experimentally mapped TSSs reflect local structural traits, which can be considered typical features of CPs. These findings have been corroborated by several other works [7-9], illustrating that profiles of physic- 
ochemical properties indeed reveal a TSS specific signal in several eukaryotic genomes.

Despite these recent works into non-motif-based descriptors of CPs, computational methods of promoter identification principally rely on conserved cis-acting sequence motifs (in many cases, $\mathrm{CpG}$ islands) as descriptors. The extent of this preference is evident from a recent review of promoter prediction programs (PPPs) [10] where all of the eight programs examined use some direct motif/CpG based feature. While in some cases this approach has proven to be very effective $[11,12]$, it is only applicable when the CPs in question are associated with clearly defined sequence elements. In several studies, however, DNA physicochemical properties were incorporated into predictor mechanisms. In the case of McPromoter by Ohler et al. [13], the incorporation of a single such parameter into their prediction framework reduced false positive predictions of Drosophila melanogaster CPs. More recently, it was shown that by identifying peaks in profiles of DNA structural properties along eukaryotic genomic sequences, CPs could be predicted more accurately than with other PPPs [9]. Furthermore, a PPP was recently developed that used six different physical DNA properties to distinguish between CPs and other DNA sequences, and was shown to outperform 'traditional' PPPs across diverse datasets from eukaryotic genomes [7].

Our interest in prediction methods based on physicochemical properties stems from our studies of promoter regions in $P$. falciparum, the most virulent agent of human malaria, causing millions of deaths globally every year [14]. This parasite is characterized by a complex life cycle that involves two hosts (an invertebrate - mosquito - and a vertebrate - in the case of $P$. falciparum, human) and several morphologically different stages. Such complexity implies dynamic transcriptional control of gene regulation; however, very little is known about the transcriptional mechanisms of this parasite (see reviews in $[15,16])$. While recent studies have begun to shed some light on these processes through the identification of specific transcription factors and their binding sites [17], the general paucity of information coupled with the exceptionally AT-rich genome [18] mean that computational techniques developed for other genomes are of limited use. In fact, the only PPP that has been specifically applied to the $P$. falciparum genome [9] showed poor performance, prompting the authors to suggest that a bespoke solution was required for this organism.

In the present work, we used DNA physicochemical properties to construct profiles of $P$. falciparum CPs around experimentally determined TSSs in the FULL-Malaria database [19]. We observed characteristic maxima/minima in these profiles at the TSS, confirming previous results with similar parameters in other eukaryotes [6,9]. Furthermore, signals around TSSs allowed us to propose that the actual CP occupies a small region from -35 to +1 nucleotides, as in other eukaryotic genomes. Since these signals are extremely weak and obscured by noise when examined on an individual sequence basis, we have developed a predictor based on an ensemble of support vector machines (SVMs; the Malarial Promoter Predictor (MAPP)) that can identify P. falciparum $\mathrm{CP}$ regions on the basis of their distinct physicochemical properties.

This is the first time that a computational method has successfully been used to identify TSSs in this genome. We demonstrated that MAPP not only distinguishes a large percentage of TSS positions from non-TSS sequences, but can do so with high spatial accuracy, agreeing with experimental results and representing a useful tool for experimentalists and genome annotators. MAPP predictions on a genomic scale give an insight into $\mathrm{CP}$ organization in $P$. falciparum, illustrating that physicochemical properties of the DNA are essential for promoter recognition and suggesting that TSSs occur in broad 'transcriptional start areas' rather than at precise start sites. Furthermore, particular promoter arrangements are revealed (bi-directional promoters, antisense RNA transcription, and so on) that might open novel avenues for the investigation of transcription mechanisms in this organism.

\section{Results and discussion \\ $P$. falciparum core promoter regions have typical physicochemical properties}

In order to analyze the composition and conservation of the $P$. falciparum CPs, we extracted sequences spanning 100 nucleotides upstream and 49 nucleotides downstream of each of the 3,546 experimentally mapped TSSs in the FULL-Malaria database $[19,20]$. This dataset contains at least one TSS for $27 \%$ of $P$. falciparum genes. We then aligned these sequences at the TSS and generated a position weight matrix. From this position weight matrix, we calculated nucleotide frequencies and information content at each position around the TSSs (Figure 1). We observed that thymine-adenine is the sequence highly favored at the TSS (Figure 1a), while immediately upstream, for approximately 30 nucleotides, thymine is the preferred nucleotide. Interestingly, the preference for T-A at the TSS reflects the pyrimidine-purine feature ( $\mathrm{PyPu}$ ) present at the TSS in other eukaryotes [21,22], albeit in an AT-rich form (the consensus for the PyPu feature is generally $\mathrm{C}(\mathrm{G} / \mathrm{A})$, as opposed to the strong TA preference seen here). The PyPu feature at the TSS is generally conserved across different promoter classes [23] and has been shown to be necessary for TFII $_{\mathrm{D}}$ binding in promoters lacking well defined cis-elements [24]. While this feature clearly emerges, the corresponding peak in information content ( 0.2 bits; Figure $1 \mathrm{~b}$ ) indicates that CPs in P. falciparum are characterized by weak sequence conservation. We thus hypothesized that rather than sequence elements, other factors related to the conformation of the DNA molecule may play a role in transcription initiation. This hypothesis is supported by recent evidence in other genomes [6-9]. 

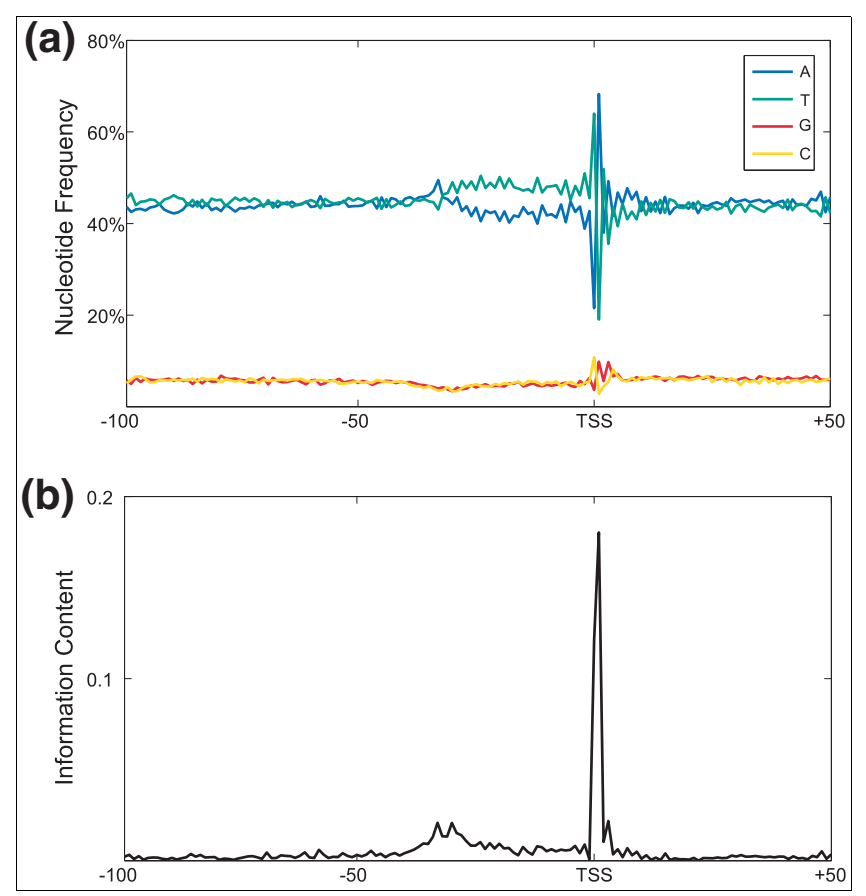

Figure I

Sequence conservation at the $P$. falciparum TSS. (a) Nucleotide frequencies in the region from -100 to +50 nucleotides around 3,546 $P$. falciparum TSSs. (b) The frequency of each position in the 150 nucleotides around aligned TSS was calculated to generate a position specific scoring matrix. The information content of each position in the matrix was calculated by $\Sigma_{i}\left(p_{i}^{*} \log _{2}\left(p_{i} / b_{i}\right)\right)$, where $p_{i}=$ frequency of nucleotide $i$ at that position and $b_{i}=$ background frequency of $i$. Background frequencies were calculated from $P$. falciparum intergenic DNA $\left(b_{A}=0.42, b_{T}=0.45, b_{G}=\right.$ $0.07, b_{c}=0.06$ ).

We used 59 experimentally determined physicochemical properties of DNA (Additional data file 1) in this analysis, along with two different measures of GC content and with the composition based LD parameter of Bultrini et al. [6]. Since these properties are based on di-, tri- and tetra-nucleotide sequences, they may reflect similar physical characteristics so that correlations among them must be considered. To do this, we performed a redundancy reduction step (see Materials and methods for details) that resulted in the removal of 28 highly correlated properties. Together with the tetra-nucleotide property (LD), this process yielded a set of 33 nonredundant physicochemical properties that was used in further work.

We generated a profile for the 33 selected properties along the 150 nucleotide sequences around each of the 3,546 experimentally mapped TSSs. We used a window size of 2, 3 or 4 nucleotides for di-, tri- or tetra-nucleotide properties, respectively, along with a shift of 1 nucleotide. The normalized average and standard deviation of the profiles are shown in Figure 2 for each of the non-redundant properties. Averaged profiles show characteristic features in a restricted area around the aligned TSSs, and in many cases a corresponding low stand- ard deviation is also observed. Even though, in nearly all cases the strongest 'signal' is seen precisely at the TSS, an additional signal with a low standard deviation is seen approximately 35 nucleotides upstream of TSSs in profiles generated using properties $14,15,19,28,32,38,39,40,43$ or 60 .

The agreement between signals from compositional and physicochemical properties paints a picture of the CP in $P$. falciparum, suggesting that, as is the case for canonical eukaryotic CPs, important features are contained in the short region between -35 nucleotides and +1 nucleotide.

\section{Support vector machine training with core promoter physicochemical profiles}

SVMs comprise a class of supervised machine learning algorithms that can, in principle, separate any two classes of objects. SVMs have been applied extensively to bioinformatic problems from analyses of microarray data to protein fold recognition (for comprehensive reviews, see [25,26]). Recently, SVMs were successfully applied to detect sequence based biological signals in the human genome, including characteristic motifs at the TSSs [27].

We decided to construct a predictor combining SVMs trained to recognize CPs in the P. falciparum genome on the basis of signals observed in the 33 physicochemical profiles. First of all, we carefully selected sequences (positive and negative data) for training and testing the SVMs. We used sequences from -100 to +49 nucleotides around each experimentally determined TSS as positive data $[19,20]$. Negative data were generated by selecting 150 nucleotide sequences from both intergenic (IG) and exonic (EX) genomic DNA (from version 2.1 of the genome). Since IG sequences may contain distal or undocumented TSSs, we used the length distribution of $5^{\prime}$ untranslated regions derived from $P$. falciparum full-length cDNAs (flcDNAs) to establish criteria for IG selection. Having observed that only $3.2 \%$ of the transcripts begin at a distance greater than 2,000 nucleotides from the closest gene, we decided to select IG sequences that were at least 2,00o nucleotides away from any annotated gene. Excessive false positive predictions is one of the greatest problems for CP predictors, and thus, we used a CP:IG:EX ratio of 1:2:2 during the training (Table 1). The remaining sequences were divided into two independent test sets, the smaller test set (Test 1) was used to find the optimal combination of SVMs for the final predictor (see below), while the larger test set (Test 2) was used to assess the final predictor.

Sequences were converted into physicochemical profiles and a SVM was trained for each of these properties. Some positions in physicochemical profiles (features) may not contribute to prediction ability and, hence, may reduce performance and increase the computational burden. For these reasons we used a wrapper-type feature selection algorithm (for details see Materials and methods) to establish positions in physicochemical profiles that best discriminate CPs from negative 

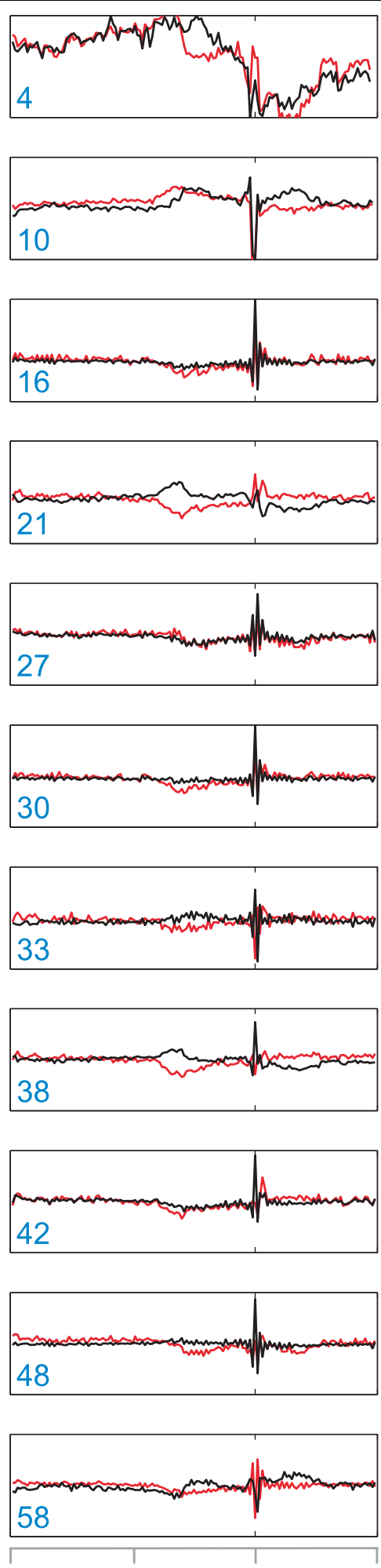

$-100$
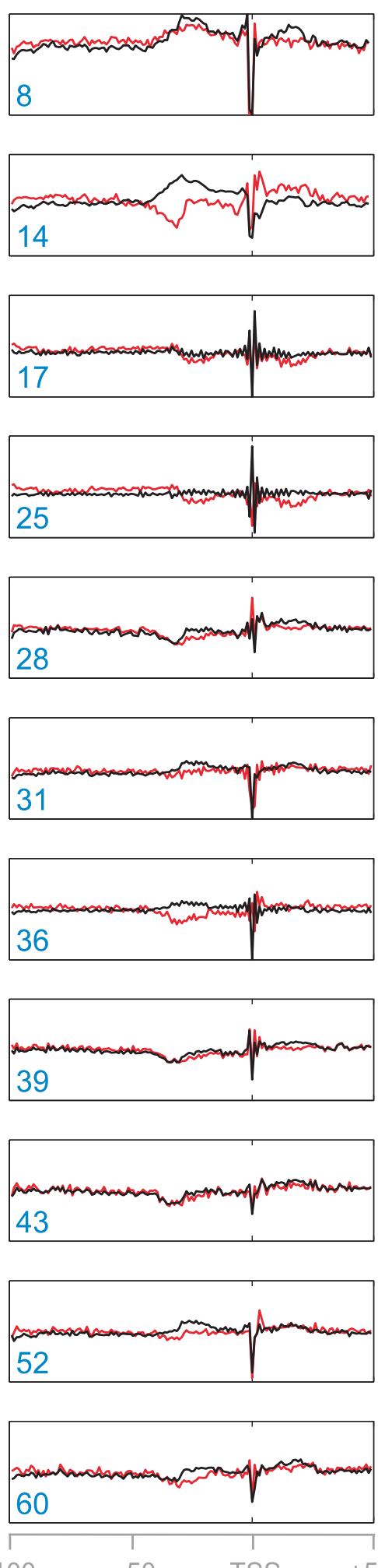
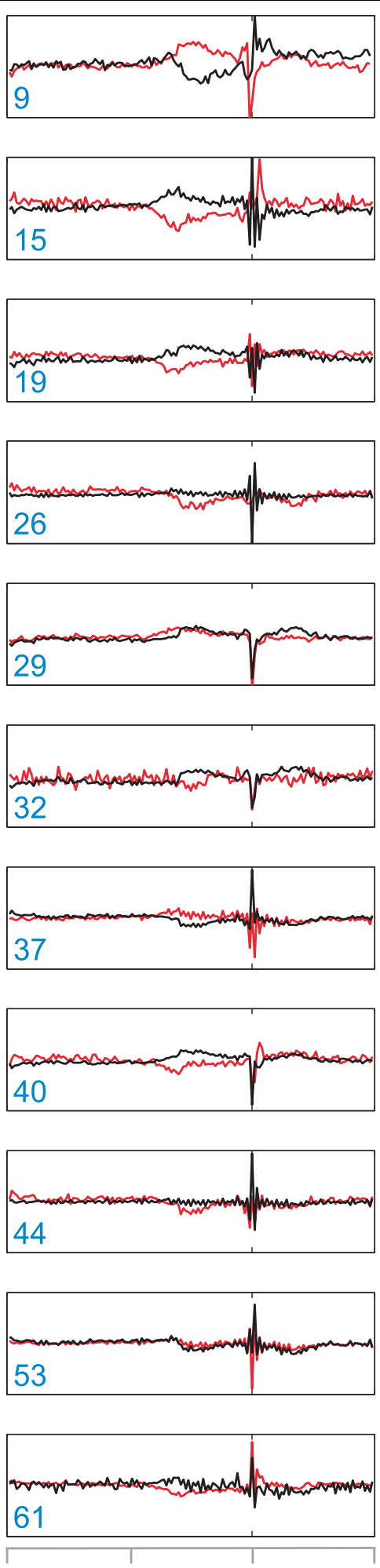

$-50-100$

Figure 2

DNA physicochemical property profiles around $\boldsymbol{P}$. falciparum TSSs. All I 50 nucleotide CP sequences were aligned at TSS positions. For each of 33 non-redundant DNA properties (identified by a progressive number; Additional data file I), the average profile over the 3,546 sequences was calculated. The average profile is shown for each profile as a black line, and the standard deviation as a red line. 
Table I

\begin{tabular}{lccc}
\hline \multicolumn{4}{l}{ Number of sequences used for SVM training and testing } \\
\hline & CP & IG & EX \\
\hline Training & 1,100 & 2,200 & 2,200 \\
Test I & 610 & 302 & 302 \\
Test 2 & 1,834 & 910 & 910 \\
\hline
\end{tabular}

$\mathrm{CP}$, number of core promoter sequences; IG, number of intergenic sequences; $E X$, number of exonic sequences.

sequences. The relevance of each position around the TSSs was evaluated, then different combinations of the most relevant ones were used to train a SVM with fivefold cross-validation. For each set of selected positions, the SVM performance was evaluated and the combination that gave optimal fivefold cross-validation accuracy during the training process was chosen (see Materials and methods; Additional data file 2). Even though this selection strategy considers positions independently, the process only results in the removal of features that have a net detrimental effect on SVM performance.

Besides reducing the computational cost and improving SVM performance, the results of this feature selection are interesting per se as they show the localized importance of each physicochemical feature around the TSS. In Figure $3 a$, the optimal set of features for training each SVM are shown (selected features are green, unselected are red). From these, a complex picture of the local physicochemical properties at the P. falciparum $\mathrm{CP}$ emerges. Some notable patterns of biological sig-

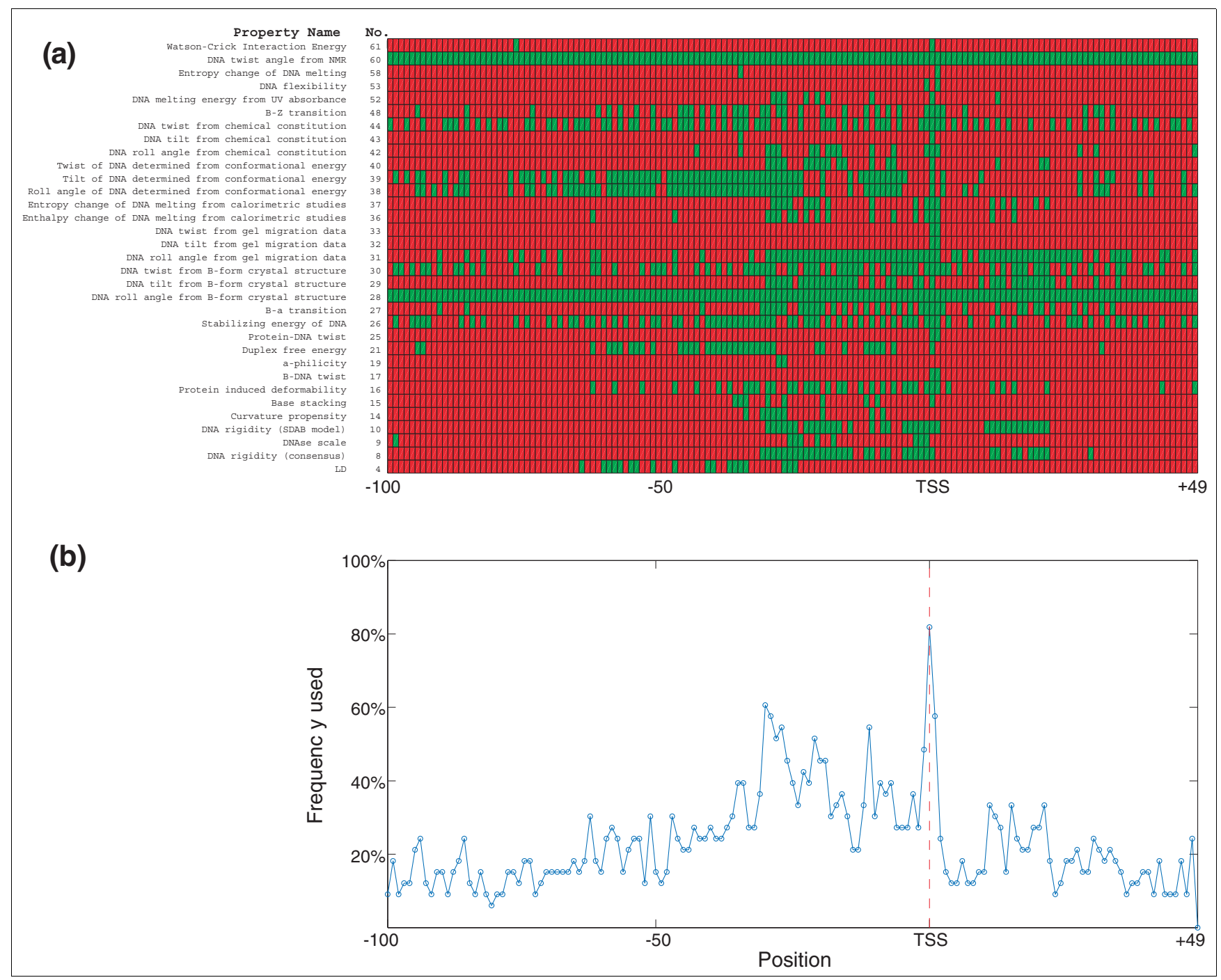

\section{Figure 3}

Frequencies of features used by SVMs for training. (a) The features used for training each SVM. Green boxes indicate features used to train an SVM with that physicochemical property. Red boxes indicate features that were not used. (b) The relative frequency with which each feature is used in SVM training highlights the most important positions for accurate SVM training. 
nificance could be identified. For example, we observed that in the region between - 31 nucleotides and the TSS, DNA rigidity is an important consideration (properties 8 and 10; 49/62 features are used). The entropy (properties 37 and 52) and enthalpy (property 36) upon 'melting' of this region are also distinctive, particularly in the 5 ' region, close to the -31 nucleotides position. These results in combination with profiles in Figure 2 suggest that while rigid, this region may be easily zipped open when required for transcription. The results for the protein-induced deformability (property 16) are also particularly interesting. Selected positions are from -64 to +30 nucleotides, suggesting that this entire region may be particularly amenable to binding of general transcription factors (such as $\mathrm{TFII}_{\mathrm{D}}$ ) that deform the DNA when they bind.

Despite the complexity of these results, when we analyzed the frequency with which each feature is used in overall SVM training (Figure $3 \mathrm{~b}$ ) a clearer pattern emerged. The most frequently used features are found precisely at the TSS (o to +1; used to train $81 \%$ and $60 \%$ of SVMs, respectively) and in the region from -35 to -20 nucleotides upstream of the TSS.

\section{Consolidation of SVMs into the MAPP}

In order to assess which of the SVMs gave the best performance, we utilized the first test dataset (Test 1). In addition to specificity and sensitivity, we also calculated the harmonic mean (F) as this measure equally weights type I (false positives) and type II errors (false negatives) (see Materials and methods). The performance for each of the 33 SVMs is reported in Table 2. The most robust single classifier $(\mathrm{F}=$ 0.52 ) is that trained with property 60 , the twist of DNA, as determined by NMR [28]. This classifier has the highest sensitivity of all SVMs (0.37), yet the specificity is somewhat low (0.97). Other SVMs, such as that trained with property 14 - AT and GC type curvature propensity [29] - correctly predict fewer promoters (sensitivity $=0.09$ ), but have a specificity of 1.0o, meaning that IG and EX sequences are never predicted as CP. Nine trained SVMs were unable to distinguish CP from negative sequences and, thus, have no predictive value (sensitivity $=0$, specificity $=1$ ). These nine SVMs were discarded and not used in subsequent steps. MAPP combines the outputs of the remaining 24 trained SVMs to give a prediction. We trained a final SVM to combine these outputs in order to derive a single MAPP score (between o and 1) for each sequence.

For each combination of the top $n$ SVMs as ranked by F-score ( $\{n \mid n \in Z, 1 \leq n \leq 24\}$; Table 2) we calculated the area under a receiver operating characteristic (ROC) curve (AUC). This is a useful single figure representation of overall performance for which random choice will yield an AUC of 0.5, while a perfect predictor will yield an AUC of 1.o. By combining individual predictions, the AUC is increased from 0.835 to 0.883 , with the maximum AUC achieved using 17 SVMs. The AUC saturates after $n=17$, yielding similar AUCs for all combinations up to the maximum of $n=24$. The cumulative effect confirms
Table 2

\begin{tabular}{|c|c|c|c|}
\hline \multicolumn{4}{|c|}{ Cross-validated SVM performances } \\
\hline Property & Sensitivity & Specificity & F-score \\
\hline 4 & 0.10 & 0.99 & 0.17 \\
\hline 8 & 0.21 & 0.99 & 0.35 \\
\hline 9 & 0.18 & 0.99 & 0.30 \\
\hline 10 & 0.28 & 0.98 & 0.43 \\
\hline 14 & 0.09 & 1.00 & 0.17 \\
\hline 15 & 0.25 & 0.97 & 0.39 \\
\hline 16 & 0.28 & 0.95 & 0.42 \\
\hline 17 & 0.00 & 1.00 & 0.00 \\
\hline 19 & 0.00 & 1.00 & 0.00 \\
\hline 21 & 0.20 & 0.97 & 0.32 \\
\hline 25 & 0.00 & 1.00 & 0.00 \\
\hline 26 & 0.34 & 0.96 & 0.49 \\
\hline 27 & 0.18 & 0.98 & 0.30 \\
\hline 28 & 0.32 & 0.96 & 0.48 \\
\hline 29 & 0.27 & 0.98 & 0.41 \\
\hline 30 & 0.30 & 0.96 & 0.45 \\
\hline 31 & 0.30 & 0.96 & 0.45 \\
\hline 32 & 0.00 & 1.00 & 0.00 \\
\hline 33 & 0.00 & 1.00 & 0.00 \\
\hline 36 & 0.24 & 0.97 & 0.37 \\
\hline 37 & 0.24 & 0.96 & 0.38 \\
\hline 38 & 0.29 & 0.96 & 0.43 \\
\hline 39 & 0.25 & 0.95 & 0.38 \\
\hline 40 & 0.12 & 0.99 & 0.21 \\
\hline 42 & 0.12 & 0.98 & 0.21 \\
\hline 43 & 0.00 & 1.00 & 0.00 \\
\hline 44 & 0.19 & 0.97 & 0.32 \\
\hline 48 & 0.33 & 0.95 & 0.48 \\
\hline 52 & 0.09 & 0.99 & 0.16 \\
\hline 53 & 0.00 & 1.00 & 0.00 \\
\hline 58 & 0.00 & 1.00 & 0.00 \\
\hline 60 & 0.37 & 0.97 & 0.52 \\
\hline 61 & 0.00 & 1.00 & 0.00 \\
\hline
\end{tabular}

The performance of each of the SVMs after cross-validated training using each individual physicochemical property of DNA.

that the physicochemical properties selected to train SVMs provide independent and complementary information on the CP in P. falciparum. To generate the final MAPP score $\left(\mathrm{M}_{\mathrm{sc}}\right)$, we chose $n=21$, a point in the middle of the optimal range.

\section{MAPP assessment}

The performance of the final predictor, MAPP, was assessed on the second test set (Test 2). First of all, we studied the distributions of $\mathrm{M}_{\mathrm{sc}}$ for $\mathrm{CP}$ and negative sequences (IG and $\mathrm{EX}$; Figure 4a). The distributions of $\mathrm{CP}$ and negative sequences only partially overlap, with most of this overlap due to IG sequences. For $\mathrm{M}_{\mathrm{sc}}$ higher than 0.05, few false positives are expected and predictions with $\mathrm{M}_{\mathrm{sc}}>0.94$ have 100\% accuracy. 


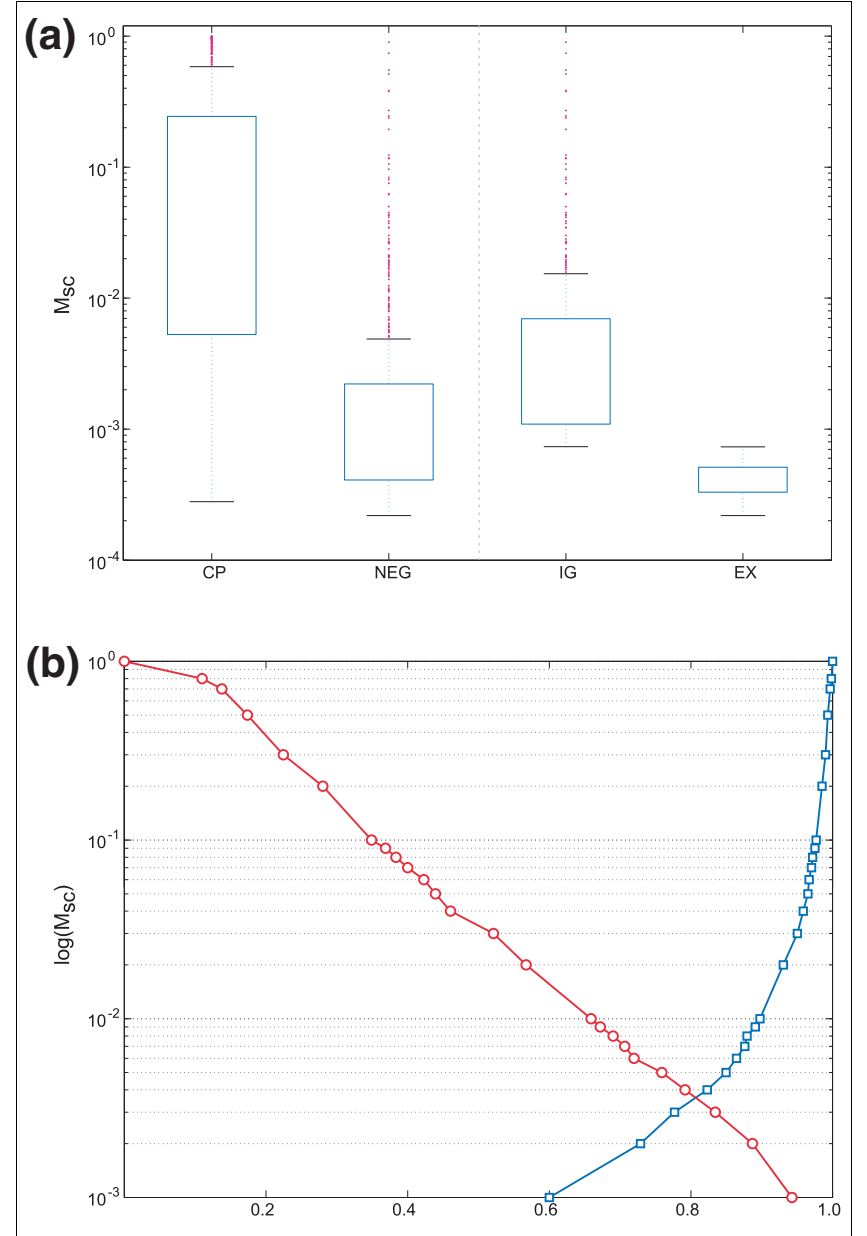

Figure 4

MAPP score distributions. (a) The distribution of MAPP scores $\left(M_{s c}\right)$ for core promoter (CP) and negative (NEG) sequences are given for the test dataset Testl. Upper and lower limits of the box represent the upper and lower quartiles of the distribution, respectively. Whiskers extending from the boxes represent the extent of the rest of the data distribution, while outliers are represented by magenta points. On the right-hand side of the dotted line is the breakdown of the NEG distribution into separate distributions for intergenic (IG) and exonic (EX) sequences. (b) The specificity (blue squares) and sensitivity (red circles) at different $M_{s c}$ thresholds.

It is more prudent to state the error rate at this threshold as $<1$ false positive per 910 nucleotides IG DNA, and $<1$ false positive per 910 nucleotides IG DNA.

A more detailed analysis reveals that a clear and highly significant ( $p<10^{-100}$, Wilcoxon rank sum test) difference is seen between the mean of the $\mathrm{CP} \mathrm{M}_{\mathrm{sc}}\left(\bar{M}_{s c}=0.19 \pm 0.30\right)$ and the mean of the negative sequence $\mathrm{M}_{\mathrm{sc}}\left(\bar{M}_{s c}=0.02 \pm 0.09\right)$. Interestingly, the three input groups (CP, IG and EX) exhibit statistically different score distributions $\left(p<10^{-100}, 3 \times\right.$ Wilcoxon rank sum test), despite not having been trained as such. This further separation of the exonic profiles is very likely due to the diverse nucleotide composition of coding and non-coding DNA in P. falciparum [18].

Quantitatively, these results are best expressed as specificity and sensitivity. We calculated these values for MAPP predictions at $30 \mathrm{M}_{\mathrm{sc}}$ thresholds (Figure $4 \mathrm{~b}$ ). At each threshold ( $t$ ), a sequence with $\mathrm{M}_{\mathrm{sc}} \geq t$ is considered a TSS prediction. For example, if we consider the most permissive criterion of $\mathrm{M}_{\mathrm{sc}} \geq$ $10^{-3}$ (any sequence with a positive $\mathrm{M}_{\mathrm{sc}}$ is considered a TSS), we achieve a sensitivity of 0.94 (red circles) and a specificity of o.6o (blue squares). By increasing the $\mathrm{M}_{\mathrm{sc}}$ threshold, the specificity increases and exceeds 0.99 at $\mathrm{M}_{\mathrm{sc}} \geq 0.6$. Notwithstanding that the CP:EX:IG ratio used in these assessments does not reflect the true ratio in the genome (where $\mathrm{CP}$ sequences would be far less frequent), the high specificity does indicate that MAPP may be well suited for genomic scale applications.

\section{Positional effect on MAPP score}

In order to assess the positional precision of MAPP, we generated a prediction for every nucleotide position in the region from -400 to +200 nucleotides around each TSS in the Test 2 dataset. At each position in the 601 nucleotide window, we calculated the average $\mathrm{M}_{\mathrm{sc}}$. We then counted the number of nucleotides adjacent to the TSS for which the $\mathrm{M}_{\mathrm{sc}}$ remained more than one standard deviation above the mean (Additional data file 3). We found this region spans 101 nucleotides almost symmetrically around the TSS. This can be considered the positional accuracy of MAPP prediction. These results, as well as being important to evaluate genome scale predictions of MAPP, are also interesting from a biological point of view. The broad distribution of high $\mathrm{M}_{\mathrm{sc}}$ in the region immediately around TSSs may be due, in part, to the presence of multiple start sites, suggesting the presence of 'transcriptional start areas' from which several transcripts arise. This is in line with the available experimental data for P.falciparum; in the three cases of finely characterized promoters [30-32] and for almost half of the genes with mapped TSSs [19], multiple start sites are observed. Furthermore, recent evidence from high throughput studies in mammalian genomes suggests that an 'area' with several TSSs dispersed over tens of nucleotides, rather than a single specific start nucleotide, is the predominant type of promoter architecture [23].

To assess the positional preferences of predictions relative to gene start codons, we generated predictions for 3,00o nucleotides upstream and 1,200 nucleotides downstream of all $P$. falciparum gene start sites. At each position we averaged the MAPP scores (blue circles in Figure 5). The MAPP score peaks in the 1,000 nucleotide region upstream of start codons. This illustrates a striking preference for strong predictions upstream of ATG start codons. Furthermore, the MAPP distribution from -3,000 nucleotides to ATG is highly correlated with the TSS distribution derived from experimental flcDNA mappings (red squares in Figure 5; Pearson correlation coefficient $=0.96$ ). Immediately 3 ' to the gene start site, there is a 


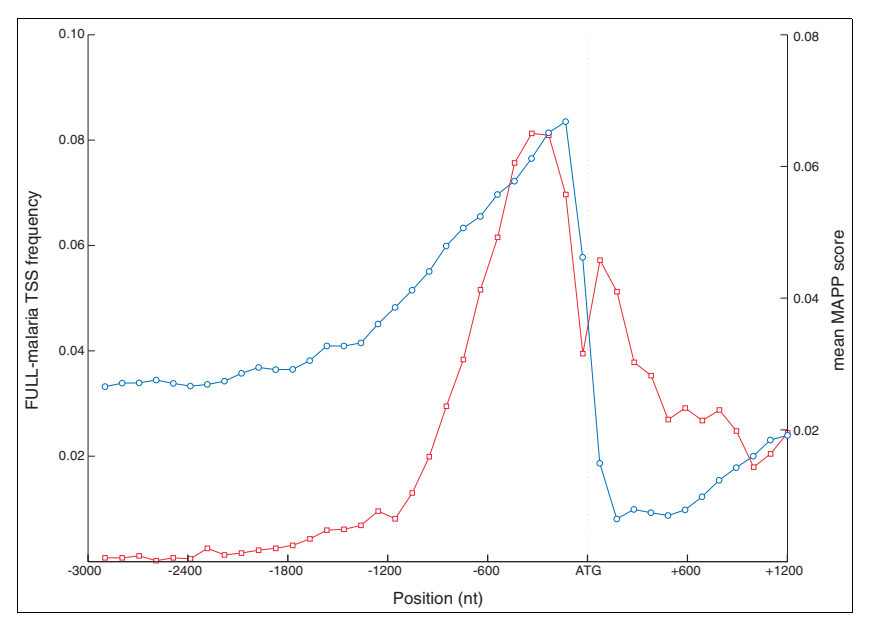

Figure 5

MAPP score distributions and comparison with experimental TSS distributions. A MAPP profile was generated for the region from 3,500 nucleotides upstream to I,200 nucleotides downstream of every gene start codon in the P. falciparum genome (v2.I.4). These MAPP profiles were aligned at the 0 position (ATG codon) and the MAPP score averaged at each position. We smoothed the average MAPP score using a sliding window of 200 nucleotides and a shift of 100 nucleotides (blue circles). The TSSs distribution was generated from the frequency of FULL-Malaria TSSs at each distance from the closest ATG codon (red squares). Multiple TSSs that mapped to the same nucleotide were considered as a single mapping.

dramatic dip in the MAPP score, confirming that MAPP makes very few TSS predictions in exonic regions.

When predictions are performed on large genomic sequences, MAPP cannot assign predictions to one strand or another. In fact, we observe very similar predictions on both DNA strands but shifted by approximately 40-50 nucleotides from each other (the correlation coefficient between the plus and minus strand profiles for chromosome 14 rises from 0.33 to 0.56 if we shift one of the profiles by 50 nucleotides). As previously shown, those positions in the SVM input vectors that are most discriminative for classifying training sequences are between -35 nucleotides and +1 nucleotide. When this region of an input vector overlaps with a strong promoter signal (that is, 35 nucleotides to +1 nucleotide around a true TSS), a high $\mathrm{M}_{\mathrm{sc}}$ is output at the TSS (position o nucleotides; for a detailed schema, see Additional data file 4). However, if the overlap is in the reverse orientation (that is, from +1 to -35 on the opposite strand), a strong, similar $\mathrm{M}_{\mathrm{sc}}$ will result for a nucleotide at the other extreme of this window (position -34 nucleotides). Other, weaker signals (from -50 to +25 nucleotides) account for the variability of the shift size observed between the two profiles. In subsequent analyses, unless otherwise stated, we consider only the MAPP predictions on the same strand as the gene of interest.

\section{Evaluation with EGASP criteria}

The Encode Genome Annotation Assessment Project (EGASP) established a set of standard criteria by which the performance of a PPP can be assessed (see Materials and methods for details) [33]. This assessment was important to give a true reflection of MAPP performance on a genomic scale, where the CP:EX:IG ratio is very different to that used in the SVM training/test processes.

For each gene with an upstream TSS in the Test 2 dataset, we constructed a MAPP profile from the position of the most upstream TSS to the downstream gene stop codon. MAPP predictions were then clustered at different $\mathrm{M}_{\mathrm{sc}}$ thresholds ( $t$; for details, see Materials and methods). This simplified each profile into a series of single point predictions (each cluster center is a prediction). In previous studies on other genomes, a maximum allowed distance of \pm 500 or \pm 1 ,000 nucleotides between true and predicted TSSs has been commonly used [33]. Given the relative compactness of the $P$. falciparum genome, we decided to consider only maximum distances $(w)$ of \pm 50 nucleotides and of \pm 100 nucleotides. Each analysis was thus extended upstream of the 5 ' TSS by $w$ nucleotides to allow for predictions that fall in this region. In addition to the positive predictive value (PPV) and sensitivity, we also calculated the harmonic mean (F). F equally weights the PPV and sensitivity, ranging from 1 (best performance) to $o$ (worst performance), and hence is a useful measure to assess overall predictor performance.

As expected, the MAPP performance was better at each $t$ cutoff when we used the \pm 100 nucleotide window size (second column in Table 3,). Irrespective of which window size was used, a reduction in the clustering threshold reduced the PPV and increased the sensitivity. In general, it also reduced the Fscore, illustrating that the PPV cost outweighed the sensitivity benefit at lower thresholds. We determined that the optimum MAPP clustering threshold as judged by F-score was $\mathrm{M}_{\mathrm{sc}}=1.0$ when using a \pm 50 nucleotide error window $(\mathrm{F}=0.40, \mathrm{PPV}=$ 0.72 , sensitivity $=0.28$ ) and Msc $\geq 0.9$ when using $\mathrm{a} \pm 100$ nucleotide window $(\mathrm{F}=0.51, \mathrm{PPV}=0.54$, sensitivity $=0.49)$.

Table 3

MAPP performance by EGASP criteria

\begin{tabular}{lcccccc}
$t$ & $\mathrm{Sn}_{50}$ & $\mathrm{PPV}_{50}$ & $\mathrm{~F}_{50}$ & $\mathrm{Sn}_{100}$ & $\mathrm{PPV}_{100}$ & $\mathrm{~F}_{100}$ \\
\hline $\mathrm{I} .0$ & 0.28 & 0.72 & 0.40 & 0.36 & 0.80 & 0.49 \\
$\geq 0.9$ & 0.34 & $0.4 \mathrm{I}$ & 0.37 & 0.49 & 0.54 & $0.5 \mathrm{I}$ \\
$\geq 0.8$ & 0.35 & 0.35 & 0.35 & 0.52 & 0.46 & 0.49 \\
$\geq 0.7$ & 0.37 & 0.32 & 0.34 & 0.55 & 0.43 & 0.48 \\
$\geq 0.6$ & 0.37 & 0.29 & 0.32 & 0.56 & $0.4 \mathrm{I}$ & 0.47 \\
$\geq 0.5$ & 0.37 & 0.27 & $0.3 \mathrm{I}$ & 0.57 & 0.38 & 0.46 \\
$\geq 0.4$ & 0.36 & 0.24 & 0.29 & 0.57 & 0.36 & 0.44 \\
$\geq 0.3$ & 0.35 & 0.22 & 0.27 & 0.59 & 0.34 & 0.43 \\
\hline
\end{tabular}

The performance of the MAPP was assessed using the criteria designed for the EGASP promoter prediction workshop. Each analysis was run with a TP window acceptance size $(w)$ of \pm 50 nucleotides or \pm 100 nucleotides. $t$, MAPP score clustering threshold; $\mathrm{PPV}_{w}$, positive predictive value; $\mathrm{Sn}_{w}$, sensitivity; $\mathrm{F}_{w}$, harmonic mean. 
In addition, if clusters are derived from only MAPP predictions with $\mathrm{M}_{\mathrm{sc}}=1$, the PPV at each window size is $>0.7$ $\left(\mathrm{PPV}_{50}=0.72 ; \mathrm{PPV}_{100}=0.80\right)$. As a result of these high PPVs, we can have a very high confidence in such MAPP predictions on genomic scale as they guarantee a very low number of false positive predictions. It should also be noted that we probably underestimated MAPP performance in this evaluation. Specifically, our evaluation over-counts false positive predictions as the FULL-Malaria database does not provide a complete representation of TSSs for a gene. This is evidenced by the fact that $73 \%$ of $P$. falciparum genes do not have a 5 ' mapped TSS. Furthermore, several studies have identified TSSs that are absent from this dataset [30,31].

From the $\mathrm{M}_{\mathrm{sc}}$ distributions in Figure 4a., we would have expected very few TSSs to have a MAPP score $\geq 0.6$ (specificity $=0.17$ ). Apparently, this is in contrast to the MAPP specificity established with EGASP criteria (specificity $=0.37$ ). This can be explained by the imprecision of flcDNA mappings or by the presence of more TSSs than we know of. flcDNAs are generated by a system that also has an implicit error. It has been shown that $7.2 \%$ of TSSs derived from flcDNA in the Database of Transcriptional Start Sites (DBTSS) were more than 100 nucleotides distant from equivalent mappings in the Eukaryotic Promoter Database (EPD) [34].

We also compared the performance of MAPP with the only other PPP that can be justifiably applied to the $P$. falciparum genome (EP3) [9]. EP3 is, however, known to perform relatively badly in this organism compared to others. We confirmed that $\mathrm{EP}_{3}$ was not effective at identifying promoters at either window size ( \pm 50 and \pm 100 nucleotides) as in both cases it yielded PPV, sensitivity and F-scores below 0.02 .

\section{Validation with independent experimental data}

We performed some independent analysis of the quality of our predictions with data not derived from the FULL-Malaria database. In this way, we could also assess the empirical usefulness of our predictions on a gene-by-gene basis. We identified independently mapped TSSs in the literature and selected the upstream regions of three representative cases for this validation (the others are illustrated in Additional data file 5). For each nucleotide in the selected regions, a MAPP score was calculated and predictions are shown as a plot along the genomic sequences (MAPP profile).

\section{PFII_0009 (rifin)}

The upstream region of the rifin gene PF11_ooog was recently characterized experimentally [31]. In this work, TSSs were mapped using 5' RLM-RACE and it was shown that transcription initiates from three positions in a 47 nucleotide window (-198, -216 and -245 nucleotides; black arrows in Figure $6 a)$. The MAPP profile peaks in the regions around all three mapped TSSs, with maximum $\mathrm{M}_{\mathrm{sc}}\left(\mathrm{M}_{\mathrm{sc}}=1\right)$ at the locations of TSSs. Furthermore, this region around the known TSSs is the only predicted putative $\mathrm{CP}$ upstream of this gene as there are no further peaks in the MAPP profile (with $\mathrm{M}_{\mathrm{sc}}>0.2$ ) for $>10,000$ nucleotides. In this case, MAPP gives a very clear indication of where transcription of this gene begins.

PFI3_00II (pfg27/25)

The region incorporating the gametocyte specific gene $p f g 27 /$ 25 was chosen for analysis as the 5 ' region of this gene has been characterized in detail experimentally [32,35]. TSSs were identified by primer extension at $-389,-394,-405$ and 413 nucleotides from the ATG (black arrows in Figure 6b). Furthermore, multiple TSSs from the FULL-Malaria database are found at positions ranging from -48 to -414 nucleotides ($48,-53,-148,-151,-267,-394,-403,-411,-413$, and -414 nucleotides; blue arrows in Figure 6b). The majority of transcripts (11 of 20) start in the region from -394 to -414 nucleotides, and seven of these map precisely to -413 nucleotides. The MAPP profile has a broad peak in the region from -376 to -501 nucleotides, which incorporates the principal site of agreement between the two experiments quoted above $(-413$ nucleotides). In fact, the multiple peaks in the -394 to -423 nucleotide region with $\mathrm{M}_{\mathrm{sc}}=1$ are in agreement with the multiple observed TSSs between these loci.

Transcripts starting from the region beyond the most upstream TSS (-414 nucleotides) were also infrequently observed in primer extension experiments (P Alano, personal communication). In these cases, primer extension and identification of large transcripts was hindered by the long unstable stretches of poly $(\mathrm{dA})$ and poly $(\mathrm{dT})$ in this region. The continuation of high scoring MAPP predictions between -424 and 493 nucleotides may be explained by this phenomenon.

The series of strong sharp prediction peaks further upstream are in a region with high AT content and a highly repetitive structure. The MAPP profile in this region is certainly interesting; however, practical difficulties mean that we have very little experimental data for this region and no mapped TSSs are known. While interesting, however, none of the peaks have $\mathrm{M}_{\mathrm{sc}}>0.8$.

\section{PFI4_0323 (pfcam)}

Previously, 47 TSSs were mapped by 5' RLM-RACE in the first 172 nucleotides upstream of the calmodulin gene (PF14_o323; black arrow in Figure 6c) [30]. Only 40 out of 93 transcripts were found to be correctly spliced, of which 36 originated from TSSs between the -90 and -172 nucleotides positions. On the contrary, un-spliced transcripts were shown to predominantly originate from the first 90 nucleotides upstream of the ATG codon and were shown to represent a very small fraction of the total mRNA pool.

We found that the strongest MAPP predictions overlap with the TSSs from which correctly spliced transcripts originate and that no MAPP peaks are found in the region immediately upstream of the gene start site. The MAPP profile between 150 and -200 nucleotides contains several high confidence 


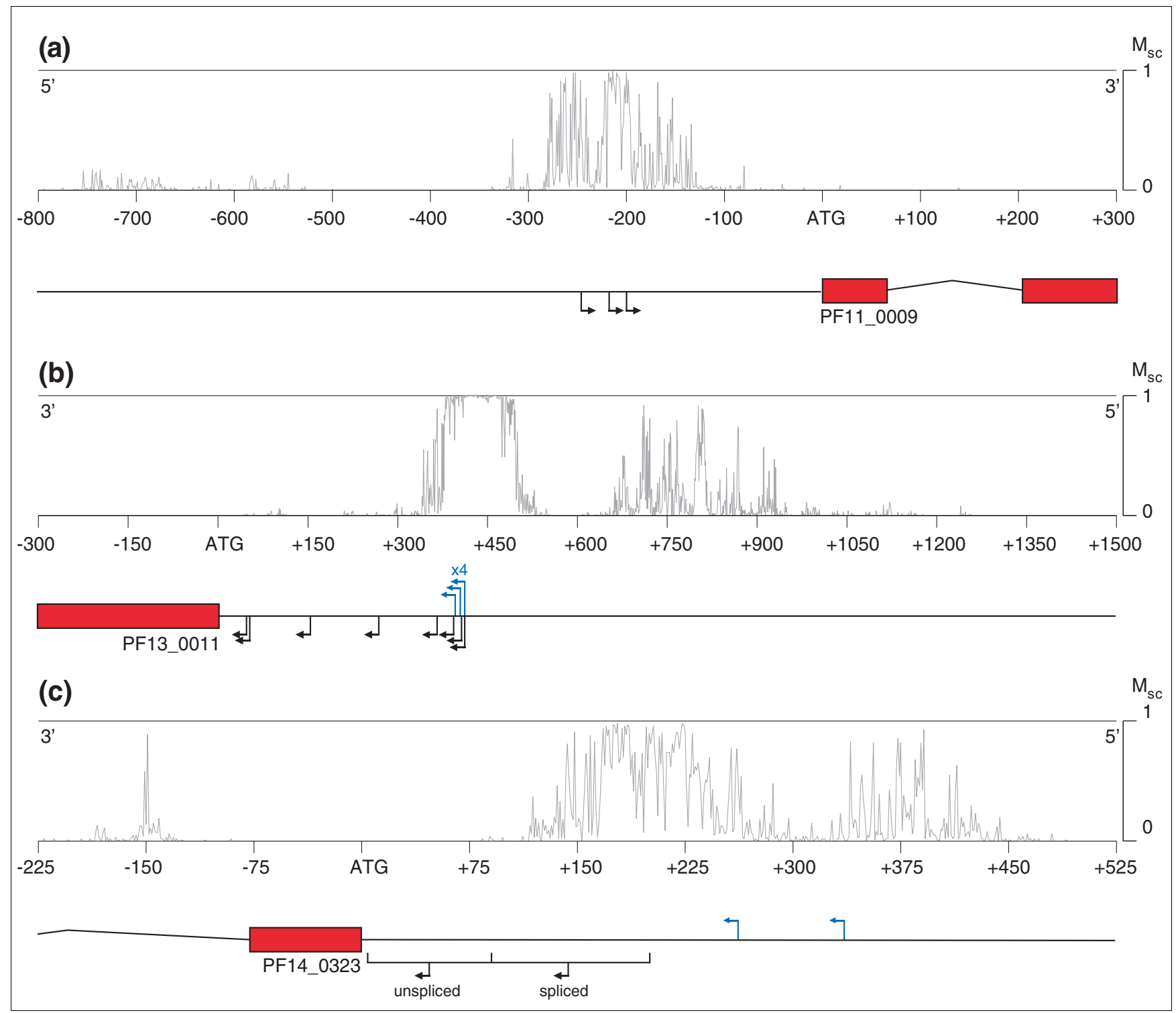

Figure 6

TSS predictions are consistent with independent experimental data. MAPP predictions for the same strand as the studied gene are plotted above the genome annotation. (a) PFII_0009; (b) PFI3_00I I; (c) PFI4_0323. The MAPP profile ranges from 0 to I (maximum). Red rectangles represent genes and arched lines represent introns. The genome is represented by the black line upon which each gene is centered. Blue arrows above the genome line represent TSSs from the FULL-Malaria database, while black arrows below the genome line are those that have been identified in other studies. Numbers above these arrows are the number of multiple TSS that could not easily be distinguished at the scale with individual arrows. In all cases, only one DNA strand is shown and directionality can be inferred from the direction of TSS arrows. The scale is given between the genome and the MAPP profile and is zeroed at the translation start site of the gene. In (c), the combined regions represented by the parentheses contain 47 individual TSSs. Those TSSs between the start codon and -80 nucleotides predominantly give rise to unspliced transcripts, while those in the region further upstream (to - 172 nucleotides) give rise to correctly spliced mRNA.

predictions with $\mathrm{M}_{\mathrm{sc}} \geq 0.97$ (151, 155, and 199 nucleotides). The MAPP profile suggests that a broad promoter is present in the region where transcription can start from several points.

Interestingly, the TSSs determined by Polson and Blackman [30] do not correspond with those present in the FULL-
Malaria database (-260 and -334 nucleotides; blue arrows in Figure $6 \mathrm{c}$ ). The MAPP profile adjacent to the TSS at -334 nucleotides indicates that a $\mathrm{CP}$ may be present in this region (peaks between -320 and 370 nucleotides), illustrating that MAPP predictions can help to consolidate and explain conflicting experimental data. These data suggest that several transcription start areas may be present upstream of this 
gene. Upstream of the illustrated region, there are no further MAPP predictions with $\mathrm{M}_{\mathrm{sc}}>0.85$ for greater than 2,000 nucleotides (in the upstream region of the adjacent gene).

\section{MAPP as an annotation tool}

We generated MAPP predictions for every nucleotide position in the $P$. falciparum genome (v2.1.4) to demonstrate some applications of MAPP on a genomic scale. In this section we discuss, on the one hand, several examples where MAPP gives us an insight into the underlying biology, and, on the other hand, some cases where the underlying biology can explain some unusual MAPP predictions. In all cases, we illustrate the forward strand prediction in green and the reverse strand prediction in black.

\section{Application I: bidirectional core promoters}

A bidirectional promoter is defined as the region (shorter than 1,000 bp) between two neighboring genes that are transcribed in opposite directions. Although these promoters have been described in human and other species, little is known as yet about their role and structure. Some correlations have, however, been found between bidirectional promoter regulated genes and human diseases [36] and there is also evidence that genes under such control may be co-regulated [37]. We identified 220 bidirectional promoter regions in the genome of $P$. falciparum, with a further 249 pairs of genes separated by less than $1,500 \mathrm{bp}$. Here we present two examples with diverse TSS arrangements as predicted by MAPP. While we usually consider just the MAPP profile on the strand containing the gene of interest, here we must analyze profiles on both strands to deduce the promoter structure.

The MAL8P1.15 and PFO8_oo11 genes are located on opposite DNA strands and share $692 \mathrm{bp} 5$ ' to their gene start sites (Figure $7 \mathrm{a}(\mathrm{i})$ ). Peaks in the MAPP profiles are found just upstream of each of the gene start codons (MAL8P1.15 from 96 to -156 nucleotides; PFO8_oo11 from -83 to -162 nucleotides], suggesting that two distinct CPs are present in this region. As a result of MAPP prediction symmetry, we cannot establish which putative promoter is associated with which gene; however, expressed sequence tag (EST) assembly data from PlasmoDB [38] confirm that these genes are divergently transcribed. Furthermore, their transcripts arise from the regions predicted by MAPP, which suggests that the promoter for each gene is that closest to it (data not shown).

A different arrangement is found for the MAL8P1.101 and $M A L 8 P 1.102$ genes (Figure 7a(ii)). Here, the MAPP profile has a single major peak in the center of the region (MAL8P1.101 from -558 to -585 nucleotides; MAL8P1.102 from -435 to -466 nucleotides) with a minor peak just upstream in the case of the forward strand. As in the previous example, EST data show that these genes are divergently transcribed, and agree with the MAPP profile. While predictions on complementary strands contribute to the promoter signal, clearly the structure of this region is such that a single $\mathrm{CP}$ is shared by the two genes.

These two diverse arrangements correspond with two different biological phenomena. In the first case MAL8P1.15 and PFo8_oO11 have similar temporal expression patterns as determined from microarray experiments [39,40], while in the second case the two genes are not co-transcribed. One may speculate that this is due to the presence of a single initiation site in the latter example, which may exclude the possibility of contemporary transcription of these two genes by separate DNA polymerase complexes. This simple example illustrates how MAPP can be used to distinguish between different architectures of $P$. falciparum bi-directional promoters. We envisage that such information may be exploited to devise novel avenues for future research on gene transcriptional control in this organism.

\section{Application 2: unusual MAPP predictions illustrate biological phenomena}

When screening at a genomic scale we see many unusual MAPP predictions, which is to be expected given our dearth of knowledge of regulatory regions in P. falciparum. We wished to assess if some of these unusual predictions were indicative of transcription-related phenomena.

The first example concerns the var gene introns. The multigene var family contains approximately 60 members and is of particular interest because var genes are regulated by a complex mechanism of allelic exclusion (only one of these genes is expressed at a time, while the rest are repressed) [41,42] involving interaction between upstream and intronic elements [43]. The MAPP profile in all var introns is very distinctive and is conserved among all members of the family (examples for six var genes are shown in Figure $7 \mathrm{~b}(\mathrm{i})$ ). Within the var intron, three different zones can be distinguished by the MAPP profile (Figure $7 \mathrm{~b}(\mathrm{ii})$ ). An unusually large region of high $\mathrm{M}_{\mathrm{sc}}$ is evident in the central part of the var intron occupying a region of approximately 400-600 nucleotides. The surrounding region is characterized by very low values for $\mathrm{M}_{\mathrm{sc}}$. This agrees with experimental evidence that has shown that the var intron indeed contains a promoter in the central region [42,44], and that transcripts of different lengths arise from multiple TSSs [42].

A second example concerns genomic regions that are located downstream with respect to genes on either strand (that is, between convergently transcribed genes). These regions are not expected to contain a classical promoter; however, in many cases, strong MAPP predictions are observed. We hypothesized that such predictions may be explained by antisense transcription, a phenomenon shown to be relatively frequent in P. falciparum [45-47]. As antisense transcripts are transcribed by the RNA polymerase II complex in $P$. falciparum [47], it is logical to expect TSSs for antisense transcripts to be identified by MAPP even if antisense TSSs were 


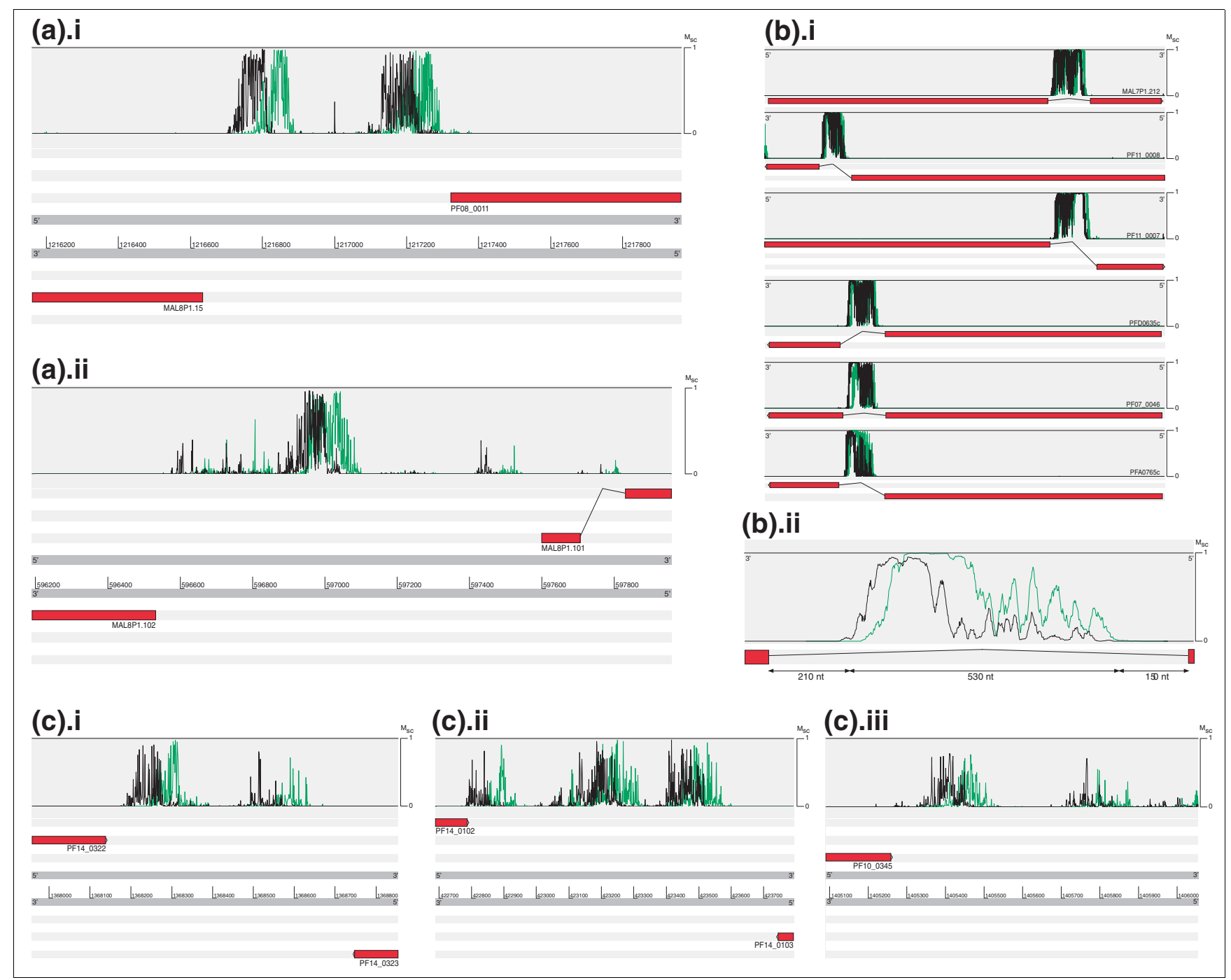

Figure 7

MAPP as an annotation tool. All figures are screenshots taken from Artemis [56]. Gene names and the $M_{\mathrm{sc}}$ scale have been added manually and all annotated exons have been colored red. MAPP profiles in green represent forward strand predictions while those in black represent reverse strand predictions. (a) MAPP profiles for both DNA strands in two bidirectional promoter regions: (i) between the divergently transcribed MAL8PI.I5 and PF08_00II genes; (ii) between the divergently transcribed MAL8PI.I0I and MAL8PI.I02 genes. (b) (i) MAPP profiles of 6 var gene introns; (ii) detailed MAPP profile of the 89I nucleotides intron of var gene PFA0765c. (c) MAPP profiles of the 3' regions of three genes identified as having anti-sense transcripts in [45]. These MAPP predictions are on the antisense strand for each gene: (i) PFI4_0323: cam; (ii) PFI4_0I02: rap-I; (iii) PFI0_0345: msp-3.

not part of the training set. Figure $7 \mathrm{c}$ shows the MAPP profiles in the 3 ' regions of the three genes shown by Patankar et al. [45] to produce antisense transcripts (PF14_o323 (cam), PF14_O1O2 (rap-1) and PF1O_o345 ( $m s p-3)$ ). In the case of cam (Figure $7 \mathrm{c}(\mathrm{i})$ ) and rap-1 (Figure $7 \mathrm{c}(\mathrm{ii})$ ) genes, the $3^{\prime}$ region is shared with a gene on the opposite strand and strong MAPP signals are seen. For the $m s p-3$ (Figure $7 c($ iii)) gene, there are 2,000 nucleotides between the stop and the start codons of the two adjacent genes on the same strand. Here, a MAPP peak is found approximately 150 nucleotides after the stop codon.
In addition, we examined the occurrence frequency of 3 ' predictions in the whole genome. We found that $40 \%$ of genes had a MAPP prediction with a $\mathrm{M}_{\mathrm{sc}} \geq 0.98$ in the 1 , 000 nucleotides 3 ' to the annotated stop codon. Intriguingly, the distribution of these MAPP peaks is not random as analysis of the mean MAPP profile showed a peak between 400 and 600 nucleotides from the stop codon, similar to that observed in $5^{\prime}$ predictions. Ultimately, the presence of strong MAPP predictions in the 3 ' regions suggests that such signals may indeed identify the location of TSSs for antisense transcripts. 


\section{Conclusion}

The identification of CPs in eukaryotic genomes is one of the major challenges for computational biologists due to the complex nature of promoters and high variability in their organization and sequences. This is particularly true for the highly AT rich $P$. falciparum genome. Several studies have been carried out aimed at identifying sequence elements involved in promoter activity or to specify minimal promoter elements, but to date even the most basic details of the structure of $P$. falciparum promoters have remained elusive. In this paper, we have shown that by using physicochemical and compositional properties of the DNA molecule, it is possible to train a predictor (MAPP) that identifies potential transcription start sites in the genome of $P$. falciparum. We showed that MAPP predictions strongly agree with available experimental data for well studied promoters and reveal diverse arrangements and architectures for promoter regions. Our results add to a growing body of evidence that a physicochemical code for eukaryotic genomes plays a crucial role in $\mathrm{CP}$ recognition.

\section{Materials and methods Datasets}

We mapped 5' RACE ends for $P$. falciparum full length cDNAs [19] were mapped to the genome (v2.1) by the EST mapping algorithm Exonerate [48] using a score cutoff of 500. We mapped 9,269 out of 12,083 5'fragments to genomic locations. All 5' fragments that mapped to within 50 nucleotides of the 5 ' clone end were considered valid mappings. TSSs were defined as the most 5 ' base from each clone that mapped to the genome. Sequences that matched partially with the genomic sequences, those for which the 5 ' nucleotide mapped within an exon or intron, and those with duplicate TSS positions were disregarded. The region from -100 nucleotides to +49 nucleotides around each TSS was extracted, yielding a dataset of 3,553 non-redundant sequences. Sequences containing wild card characters were disregarded to leave 3,546 sequences.

Our negative dataset consisted of exonic sequences and intergenic sequences. Sequences were downloaded from PlasmoDB v5.4 [38]. The exonic dataset was generated from all $P$. falciparum exons of length longer than 150 nucleotides. For the intergenic sequence dataset, we examined the distribution of 5 ' untranslated region lengths (calculated from TSSs mapped previously with exonerate) and used a value above the 95th percentile (2,000 nucleotides) as a minimum distance in which TSSs are unlikely to be present. We then extracted all intergenic regions from the genome that were more than 2,000 nucleotides distant from any coding region in either direction on either strand. For both the exonic and intergenic datasets, all possible contiguous 150 nucleotide sequences were generated from each sequence. This gave 13,196 non-redundant exonic sequences and 3,407 nonredundant intergenic sequences. We randomly selected 3,407 exons from the exonic dataset and the rest were disregarded.

\section{Selection of physicochemical DNA properties}

Values for physicochemical DNA properties were obtained from the International Centre for Genetic Engineering and Biotechnology [49,50] and from the Berkeley Drosophila Genome Project [51]. In order to eliminate redundancies, we calculated correlations between properties. To allow comparison of di- and tri-nucleotide properties, we considered the mean value of each property for all possible hexamers. Using these 6-mer values, we then calculated correlation coefficients $(r)$ between all properties to generate a distance matrix (Additional data file 6). For each row (property) of this matrix, the average absolute $r(|\bar{r}|)$ was calculated. The matrix rows were then ordered by ascending $|\bar{r}|$. Starting from the first row (that with the lowest $|\bar{r}|$ ), we removed all those properties for which the correlation coefficient with this property was greater than 0.9. We then iterated for each row, until no further correlations greater than 0.9 were found. This resulted in the removal of 28 highly correlated properties. LD parameter tetra-nucleotide values were obtained from E Bultrini (personal communication).

\section{Training/test datasets}

We used 1,100 CP, 2,200 EX and 2,200 IG sequence profiles to train each SVM. The sequences were all chosen at random. All sequences in the region from 3,800 bp downstream to 3,500 bp upstream of PF13_oo11, in the region 5,000 bp upstream of PF14_O323 and in the region from 1,921 bp downstream to 3,000 bp upstream of PF11_OOO9 were excluded from the training set. The first test dataset (Test 1) contained $610 \mathrm{CP}, 302 \mathrm{IG}$ and $302 \mathrm{EX}$ sequences and the second one (Test 2) contained 1,834 CP, 910 IG and $910 \mathrm{EX}$ sequences.

\section{Statistical quantities}

The statistical values used to assess performance throughout this paper are those used frequently in information retrieval:

$$
\begin{gathered}
\text { Sensitivity }=\frac{T P}{T P+F N} \\
\text { Specificity }=\frac{T N}{F P+T N} \\
\text { PPV }=\frac{T P}{T P+F P} \\
\mathrm{~F}=2 * \frac{(P P V * S e n)}{(P P V+S e n)}
\end{gathered}
$$

where TP, FP, TN and FN are the number of true positives, false positives, true negatives and false negatives, respectively. 


\section{SVM training and feature selection}

The libSVM library and additional tools were downloaded from [52,53]. For each sequence in each dataset (CP, EX and IG), 33 physicochemical property profiles were generated from the corresponding polynucleotide's property score at every nucleotide position in a sequence. Thus, each sequence of length $n$ and property poly-nucleotide size, $w$, was translated into a profile vector of length $(n-(w+1))$ for each property. Profiles were then normalized to the range o-1.

A wrapper-type feature selection algorithm was used for training 33 SVMs with radial bias function (RBF) kernels using each of the 33 sets of profiles. Each position (feature) in a set of profiles was ranked individually according to a score as described in [54] and a series of trial feature sets were generated by iteratively halving the number of top features used. This method does not account for interdependencies between features. This led to $\log _{2}(n)$ trial feature sets, where $n$ is the total number of features. For each trial set of features, an exhaustive search for the optimal parameters of the RBF kernel was performed using fivefold cross-validation. We chose the feature combination that used the fewest features, yet was within $1 \%$ of the highest obtained accuracy. The feature selection was carried out by a modified version of the fselect.py script downloaded with the libSVM tools.

Each trained SVM outputs a score between 0 and 1 . We used the outputs of the top $n$ best performing SVMs $\{n \mid n \in Z, 1 \leq n$ $\leq 24\}$ as input vectors for a final RBF kernel SVM. Fivefold cross-validation was used to determine the optimal parameters for training and the -b option was used to provide probability estimates as output. A flow diagram illustrating the key points of the training and prediction processes is given in Additional data file 2.

\section{MAPP positional bias}

We generated a prediction for every nucleotide position from - 400 to +200 nucleotides around each TSS in the Test 2 dataset. At each position in the 601 nucleotide window, we calculated the average $\mathrm{M}_{\mathrm{sc}}\left(\bar{M}_{s c}\right)$ for all sequences. We then counted the number of nucleotides adjacent to TSSs for which $\bar{M}_{s c}$ remained above the mean +1 standard deviation.

We also generated a prediction for every nucleotide position from $-3,000$ to $+1,200$ nucleotides around all P. falciparum gene start sites. At each position, we calculated the average $\mathrm{M}_{\mathrm{sc}}\left(\bar{M}_{s c}\right)$ for all sequences. The TSSs distribution was generated from the 3,546 TSSs selected previously and from the distance to the closest ATG start codon for each of the FULLMalaria flcDNA ends that mapped to coding regions.

\section{MAPP evaluation by EGASP criteria}

Each gene with at least one TSS in the Test 2 dataset was examined. The region assessed ranged from 100 nucleotides upstream of the most 5 ' TSS to the gene stop codon for each gene. For each region, a MAPP score for each nucleotide was generated (MAPP profile). MAPP predictions were then clustered to allow simpler evaluation using EGASP criteria. Positive predictions were those positions that had a $\mathrm{M}_{\mathrm{sc}} \geq t$, where $t$ is the chosen threshold. Any positive predictions that were separated by $\leq 50$ nucleotides were placed in the same cluster and the mean position of each cluster was then treated as a single positive prediction. At low thresholds, this method results in decreased specificity as clusters become too broad. We thus clustered predictions at all $\mathrm{M}_{\mathrm{sc}}$ thresholds ( $t$ ) from 0.3 to 1 in increments of 0.1 .

We performed the EGASP assessment [33] on all MAPP clustering thresholds. To evaluate true positive/false positive predictions, we used the evaluation criteria devised for the promoter prediction section of EGASP. We used window sizes $(w)$ of \pm 50 and \pm 100 nucleotides to evaluate performance. The region examined for each gene was from $w$ nucleotides upstream of the most 5 ' TSS to the gene stop codon. For each test sequence, true positive, false positive and false negative predictions were calculated. A prediction was considered a true positive if found within $\pm w$ nucleotides of a known TSS. Importantly, only one true positive prediction per TSS is counted, even if multiple predictions are present. Every prediction that fell outside TSS $\pm w$ nucleotide regions was considered a false positive. We calculated the PPV, sensitivity and $\mathrm{F}$ (harmonic mean) for each $\mathrm{M}_{\mathrm{sc}}$ threshold by the formulae given previously.

EP3 predictions were generated using the downloadable Java application. We performed a whole genome prediction using the Genome size <200 Mbp setting. Each site highlighted by $\mathrm{EP} 3$ was considered a prediction.

\section{Availability}

Forward and reverse strand whole genome MAPP predictions are available online as gBrowse tracks in version 5.4 of PlasmoDB [38]. Flat files, genome-wide clustered predictions, SVM models and prediction scripts are available at [55] or upon request from the authors.

\section{Abbreviations}

AUC: area under a receiver operating characteristic curve; CP: core promoter; EGASP: Encode Genome Annotation Assessment Project; EST: expressed sequence tag; EX: exonic genomic DNA; flcDNA: full-length cDNA; IG: intergenic genomic DNA; MAPP: Malaria Promoter Predictor; $\mathrm{M}_{\mathrm{sc}}$ : MAPP score; PPP: promoter prediction program; PPV: positive predictive value; RBF: radial bias function; SVM: support vector machine; TSS: transcription start site. 


\section{Authors' contributions}

KB carried out data analysis and computational work. JW contributed TSS data and critically evaluated the manuscript. $\mathrm{KB}$ and EP participated in the design and coordination of the study, the analysis of results and the writing of the manuscript. EP conceived the study. All the authors have read and approved the final version of the manuscript.

\section{Additional data files}

The following additional data are available with the online version of this paper. Additional data file 1 is a table listing the experimentally determined DNA physicochemical properties used. Additional data file 2 provides MAPP flow diagrams illustrating the key steps in the individual SVM training/feature selection and MAPP prediction processes. Additional data file 3 provide MAPP profiles around experimentally mapped TSSs. Additional data file 4 presents MAPP predictions on both DNA strands. Additional data file 5 is the MAPP analysis around independently identified TSSs. Additional data file 6 presents correlations between DNA physicochemical properties.

\section{Acknowledgements}

The authors would like to express gratitude to Yutaka Suzuki, Hiroyuki Wakaguri and Sumio Sugano for advance access to $P$. falciparum flcDNA sequences. We also wish to thank Tomasino Pace and Clara Frontali for critical feedback during manuscript preparation and Pietro Alano, Marta Ponzi, Matt Berriman and Giulio Pavesi for helpful critiques and discussion during the course of these experiments. Finally, we wish to thank Brian Brunk, Bindu Gajria, Omar Harb and David Roos for the incorporation of our data into PlasmoDB. This work was funded by the EC Network of Excellence BioMalPar grant number LSHP-CT-2004-503578.

\section{References}

I. Cooper SJ, Trinklein ND, Anton ED, Nguyen L, Myers RM: Comprehensive analysis of transcriptional promoter structure and function in $1 \%$ of the human genome. Genome Res 2006, 16:1-10.

2. Gershenzon NI, loshikhes IP: Synergy of human Pol II core promoter elements revealed by statistical sequence analysis. Bioinformatics 2005, 21:1295-1300.

3. Schones DE, Zhao K: Genome-wide approaches to studying chromatin modifications. Nat Rev Genet 2008, 9:179-191.

4. Bird A: Perceptions of epigenetics. Nature 2007, 447:396-398.

5. Segal E, Fondufe-Mittendorf $Y$, Chen L, Thastrom A, Field $Y$, Moore IK, Wang JP, Widom J: A genomic code for nucleosome positioning. Nature 2006, 442:772-778.

6. Bultrini E, Pizzi E: A new parameter to study compositional properties of non-coding regions in eukaryotic genomes. Gene 2006, 385:75-82.

7. Goni JR, Perez A, Torrents D, Orozco M: Determining promoter location based on DNA structure first-principles calculations. Genome Biol 2007, 8:R263.

8. Florquin K, Saeys Y, Degroeve S, Rouze P, Van de PY: Large-scale structural analysis of the core promoter in mammalian and plant genomes. Nucleic Acids Res 2005, 33:4255-4264.

9. Abeel T, Saeys Y, Bonnet E, Rouze P, Van de PY: Generic eukaryotic core promoter prediction using structural features of DNA. Genome Res 2008, 18:310-323.

10. Bajic VB, Tan SL, Suzuki Y, Sugano S: Promoter prediction analysis on the whole human genome. Nat Biotechnol 2004, 22: |467-|473.

II. Reese MG: Application of a time-delay neural network to promoter annotation in the Drosophila melanogaster genome.
Comput Chem 200I, 26:5I-56.

12. Ohler U, Liao GC, Niemann H, Rubin GM: Computational analysis of core promoters in the Drosophila genome. Genome Biol 2002, 3:RESEARCH0087.

13. Ohler U, Niemann H, Liao G, Rubin GM: Joint modeling of DNA sequence and physical properties to improve eukaryotic promoter recognition. Bioinformatics 200I, I7(SuppI I):S199-S206.

14. World Health Organization: WHO (2004). The World Health Report 2004 - Changing History World Health Organization; 2004.

15. Coleman BI, Duraisingh MT: Transcriptional control and gene silencing in Plasmodium falciparum. Cell Microbiol 2008, 10:1935-1946.

16. Llinas M, Deitsch KW, Voss TS: Plasmodium gene regulation: far more to factor in. Trends Parasitol 2008, 24:55I-556.

17. De Silva EK, Gehrke AR, Olszewski K, Leon I, Chahal JS, Bulyk ML, Llinas M: Specific DNA-binding by Apicomplexan AP2 transcription factors. Proc Natl Acad Sci USA 2008, 1 05:8393-8398.

18. Gardner MJ, Hall N, Fung E, White O, Berriman M, Hyman RW, Carlton JM, Pain A, Nelson KE, Bowman S, Paulsen IT, James K, Eisen JA, Rutherford K, Salzberg SL, Craig A, Kyes S, Chan MS, Nene V, Shallom SJ, Suh B, Peterson J, Angiuoli S, Pertea M, Allen J, Selengut J, Haft $D$, Mather MW, Vaidya AB, Martin DM, et al:: Genome sequence of the human malaria parasite Plasmodium falciparum. Nature 2002, 4I 9:498-5I I.

19. Watanabe J, Suzuki Y, Sasaki M, Sugano S: Full-malaria 2004: an enlarged database for comparative studies of full-length cDNAs of malaria parasites, Plasmodium species. Nucleic Acids Res 2004, 32:D334-D338.

20. FULL Malaria Database [http://fullmal.hgc.jp/index.html]

21. Kawaji H, Frith MC, Katayama S, Sandelin A, Kai C, Kawai J, Carninci $P$, Hayashizaki $Y$ : Dynamic usage of transcription start sites within core promoters. Genome Biol 2006, 7:RII8.

22. Frith MC, Valen E, Krogh A, Hayashizaki Y, Carninci P, Sandelin A: A code for transcription initiation in mammalian genomes. Genome Res 2008, 18:1-12.

23. Sandelin A, Carninci P, Lenhard B, Ponjavic J, Hayashizaki Y, Hume DA: Mammalian RNA polymerase II core promoters: insights from genome-wide studies. Nat Rev Genet 2007, 8:424-436.

24. Gallagher PG, Nilson DG, Wong C, Weisbein JL, Garrett-Beal LJ, Eber SW, Bodine DM: A dinucleotide deletion in the ankyrin promoter alters gene expression, transcription initiation and TFIID complex formation in hereditary spherocytosis. Hum Mol Genet 2005, 14:250I-2509.

25. Noble WS: Support vector machine applications in computational biology. In Kernel Methods in Computational Biology Edited by: Scholkopf B, Tsuda K, Vert J. Cambridge, MA: The MIT Press; 2004:7|-92.

26. Noble WS: What is a support vector machine? Nat Biotechnol 2006, 24:1565-1567.

27. Sonnenburg S, Zien A, Ratsch G: ARTS: accurate recognition of transcription starts in human. Bioinformatics 2006, 22:e472-e480.

28. Uljanov N, James T: Statistical analysis of DNA duplex structural features. Methods Enzymol 1995, 26I:90-1 I5.

29. Gabrielian A, Pongor S: Correlation of intrinsic DNA curvature with DNA property periodicity. FEBS Lett 1996, 393:65-68.

30. Polson HE, Blackman MJ: A role for poly(dA)poly(dT) tracts in directing activity of the Plasmodium falciparum calmodulin gene promoter. Mol Biochem Parasitol 2005, I 4 I: I79-189.

31. Tham WH, Payne PD, Brown GV, Rogerson SJ: Identification of basic transcriptional elements required for rif gene transcription. Int J Parasitol 2007, 37:605-6I5.

32. Alano P, Silvestrini F, Roca L: Structure and polymorphism of the upstream region of the pfg27/25 gene, transcriptionally regulated in gametocytogenesis of Plasmodium falciparum. Mol Biochem Parasitol 1996, 79:207-2 17.

33. Bajic VB, Brent MR, Brown RH, Frankish A, Harrow J, Ohler U, Solovyev VV, Tan SL: Performance assessment of promoter predictions on ENCODE regions in the EGASP experiment. Genome Biol 2006, 7(SuppI I):S3-13.

34. Yamashita R, Suzuki $Y$, Wakaguri H, Tsuritani K, Nakai K, Sugano S: DBTSS: DataBase of Human Transcription Start Sites, progress report 2006. Nucleic Acids Res 2006, 34:D86-D89.

35. Olivieri A, Silvestrini F, Sanchez M, Alano P: A 140-bp AT-rich sequence mediates positive and negative transcriptional control of a Plasmodium falciparum developmentally regulated promoter. Int J Parasitol 2007, 38:299-3I2.

36. Yang MQ, Koehly LM, Elnitski LL: Comprehensive annotation of 
bidirectional promoters identifies co-regulation among breast and ovarian cancer genes. PLoS Comput Biol 2007, 3:e72.

37. Trinklein ND, Aldred SF, Hartman SJ, Schroeder DI, Otillar RP, Myers RM: An abundance of bidirectional promoters in the human genome. Genome Res 2004, I4:62-66.

38. The Plasmodium Genome Resource [http://www.plas moDB.org]

39. Le Roch KG, Zhou Y, Blair PL, Grainger M, Moch JK, Haynes JD, de I V, Holder AA, Batalov S, Carucci DJ, Winzeler EA: Discovery of gene function by expression profiling of the malaria parasite life cycle. Science 2003, 30 I:1503-1508.

40. Bozdech Z, Llinas M, Pulliam BL, Wong ED, Zhu J, Derisi JL: The transcriptome of the intraerythrocytic developmental cycle of Plasmodium falciparum. PLOS Biol 2003, I:E5.

4I. Smith JD, Chitnis CE, Craig AG, Roberts DJ, Hudson-Taylor DE, Peterson DS, Pinches R, Newbold Cl, Miller LH: Switches in expression of Plasmodium falciparum var genes correlate with changes in antigenic and cytoadherent phenotypes of infected erythrocytes. Cell 1995, 82: I0I-II0.

42. Su XZ, Heatwole VM, Wertheimer SP, Guinet F, Herrfeldt JA, Peterson DS, Ravetch JA, Wellems TE: The large diverse gene family var encodes proteins involved in cytoadherence and antigenic variation of Plasmodium falciparum -infected erythrocytes. Cell I995, 82:89-100.

43. Deitsch KW, Calderwood MS, Wellems TE: Malaria. Cooperative silencing elements in var genes. Nature 200I, 4I 2:875-876.

44. Calderwood MS, Gannoun-Zaki L, Wellems TE, Deitsch KW: Plasmodium falciparum var genes are regulated by two regions with separate promoters, one upstream of the coding region and a second within the intron. J Biol Chem 2003, 278:34I25-34I32

45. Patankar S, Munasinghe A, Shoaibi A, Cummings LM, Wirth DF: Serial analysis of gene expression in Plasmodium falciparum reveals the global expression profile of erythrocytic stages and the presence of anti-sense transcripts in the malarial parasite. Mol Biol Cell 200I, I 2:3 I |4-3 I25.

46. Gunasekera AM, Patankar S, Schug J, Eisen G, Kissinger J, Roos D, Wirth DF: Widespread distribution of antisense transcripts in the Plasmodium falciparum genome. Mol Biochem Parasitol 2004, I 36:35-42.

47. Militello KT, Patel V, Chessler AD, Fisher JK, Kasper JM, Gunasekera A, Wirth DF: RNA polymerase II synthesizes antisense RNA in Plasmodium falciparum. RNA 2005, I I:365-370.

48. Slater GS, Birney E: Automated generation of heuristics for biological sequence comparison. BMC Bioinformatics 2005, 6:31.

49. Vlahovicek K, Kajan L, Pongor S: DNA analysis servers: plot.it, bend.it, model.it and IS. Nucleic Acids Res 2003, 3 I:3686-3687.

50. plot.it Server [http://hydra.icgeb.trieste.it/dna/plot_it.html]

5I. Berkley Drosophila Genome Project [http://www.fruitfly.org]

52. Chih-Chung C, Chih-Jen L: LIBSVM: a Library for Support Vector Machines. [http://www.csie.ntu.edu.tw/ cjlin/papers/lib svm.pdf].

53. LibSVM Toolkit [http://www.csie.ntu.edu.tw/ cjlin/libsvm]

54. Chen YW, Lin C]: Combining SVMs with Various Feature Selection Strategies. In Feature Extraction: Foundations and Applications (Studies in Fuzziness and Soft Computing) I.0th edition. Edited by: Guyon I, Gunn S, Nikravesh M. Zadeh LA: New York: Springer; 2006:315-323.

55. Malaria Promoter Predictor Site [http://www.mapp-predic tor.info/downloads]

56. Rutherford K, Parkhill J, Crook J, Horsnell T, Rice P, Rajandream MA, Barrell B: Artemis: sequence visualization and annotation. Bioinformatics 2000, 16:944-945.

57. Munteanu MG, Vlahovicek K, Parthasarathy S, Simon I, Pongor S: Rod models of DNA: sequence-dependent anisotropic elastic modelling of local bending phenomena. Trends Biochem Sci 1998, 23:341-347.

58. Goodsell DS, Dickerson RE: Bending and curvature calculations in B-DNA. Nucleic Acids Res 1994, 22:5497-5503.

59. Gromiha MM, Munteanu MG, Gabrielian A, Pongor S: Anisotropic elastic bending models of DNA. J Biol Phys 1996, 22:227-243.

60. Brukner I, Sanchez R, Suck D, Pongor S: Sequence-dependent bending propensity of DNA as revealed by DNase I: parameters for tri nucleotides. EMBO J 1995, 14:1812-1818.

61. Satchwell SC, Drew HR, Travers AA: Sequence periodicities in chicken nucleosome core DNA. J Mol Biol 1986, I 91 :659-675.

62. Ornstein RL, Rein R, Breen DL, Macelroy RD: An optimized potential function for the calculation of nucleic acid interac- tion energies. Biopolymers 1978, I7:234|-2360.

63. Olson WK, Gorin AA, Lu XJ, Hock LM, Zhurkin VB: DNA sequence-dependent deformability deduced from proteinDNA crystal complexes. Proc Natl Acad Sci USA 1998 95: I I I63-I II68.

64. Gorin AA, Zhurkin VB, Olson WK: B-DNA twisting correlates with base-pair morphology. J Mol Biol I995, 247:34-48.

65. Ivanov VI, Minchenkova LE, Chernov BK, McPhie P, Ryu S, Garges S, Barber AM, Zhurkin VB, Adhya S: CRP-DNA complexes: inducing the A-like form in the binding sites with an extended central spacer. J Mol Biol 1995, 245:228-240.

66. El Hassan MA, Calladine CR: Propeller-twisting of base-pairs and the conformational mobility of dinucleotide steps in DNA. J Mol Biol 1996, 259:95-103.

67. Sugimoto N, Nakano S, Yoneyama M, Honda K: Improved thermodynamic parameters and helix initiation factor to predict stability of DNA duplexes. Nucleic Acids Res 1996, 24:4501-4505.

68. Breslauer KJ, Frank R, Blocker H, Marky LA: Predicting DNA duplex stability from the base sequence. Proc Natl Acad Sci USA 1986, 83:3746-3750.

69. Blake RD: Encyclopedia of Molecular Biology and Molecular Medicine New York: Wiley; 1996.

70. Sivolob AV, Khrapunov SN: Translational positioning of nucleosomes on DNA: the role of sequence-dependent isotropic DNA bending stiffness. J Mol Biol 1995, 247:918-931.

7I. Ho PS, Ellison MJ, Quigley GJ, Rich A: A computer aided thermodynamic approach for predicting the formation of Z-DNA in naturally occurring sequences. EMBO J 1986, 5:2737-2744.

72. Aida M: An ab initio molecular orbital study on the sequencedependency of DNA conformation: an evaluation of intraand inter-strand stacking interaction energy. J Theor Biol 1988 , 130:327-335.

73. Bansal M, Bhattacharyya D, Ravi B: NUPARM and NUCGEN: software for analysis and generation of sequence dependent nucleic acid structures. Comput Appl Biosci I995, I I:28I-287.

74. Bolshoy A, McNamara P, Harrington RE, Trifonov EN: Curved DNA without A-A: experimental estimation of all 16 DNA wedge angles. Proc Natl Acad Sci USA 1991, 88:2312-2316.

75. Calladine CR, Drew HR: Principles of sequence-dependent flexure of DNA. J Mol Biol 1986, I 92:907-918.

76. De SP, Palleschi A, Savino M, Scipioni A: Validity of the nearestneighbor approximation in the evaluation of the electrophoretic manifestations of DNA curvature. Biochemistry 1990 , 29:9269-9273.

77. Hartmann B, Malfoy B, Lavery R: Theoretical prediction of base sequence effects in DNA. Experimental reactivity of Z-DNA and B-Z transition enthalpies. J Mol Biol 1989, 207:433-444.

78. Chalikian TV, Volker J, Plum GE, Breslauer KJ: A more unified picture for the thermodynamics of nucleic acid duplex melting: a characterization by calorimetric and volumetric techniques. Proc Natl Acad Sci USA 1999, 96:7853-7858.

79. Ivanov VI, Krilov DY, Shchyolkina AK, Chernov BK, Minchenkov LE: Decimal code controlling the B to A transition of DNA. J Biomol Struct Dynamics 1995, I 2:a I02-108.

80. Lisser S, Margalit H: Determination of common structural features in Escherichia coli promoters by computer analysis. Eur J Biochem 1994, 223:823-830.

8I. SantaLucia J Jr, Allawi HT, Seneviratne PA: Improved nearestneighbor parameters for predicting DNA duplex stability. Biochemistry 1996, 35:3555-3562.

82. Sarai A, Mazur J, Nussinov R, Jernigan RL: Sequence dependence of DNA conformational flexibility. Biochemistry 1989 , 28:7842-7849.

83. Gotoh $O$, Takashira Y: Stabilities of nearest neighbour doublets in double helical DNA determined by fitting calculated melting profiles to observed profiles. Biopolymers 1980, 20:1033-1042.

84. Ulyanov NB, James TL: Statistical analysis of DNA duplex structural features. Methods Enzymol 1995, 26 I:90-120.

85. Lewis JP, Sankey OF: Geometry and energetics of DNA basepairs and triplets from first principles quantum molecular relaxations. Biophys J 1995, 69: I068-1076. 